\title{
ON THE CAPACITY OF A CONDENSER
}

\author{
G. SZEGö
}

1. Introduction. (a) Any student of electric phenomena is familiar with the Leyden jar. In its simplest form it consists of two circular cylinders, with a common axis, charged with electricity. If there is a difference in the potentials of the cylinders, then electric energy, which can be recovered at any time in the form of electric discharge, is stored up in the jar.

Let us denote the radii of the cylinders by $a$ and $b, a<b$. Disregarding the effect of the ends, the potential $u$ of the electric field between the cylinders depends only on the distance $r$ from the axis, and so it must have the form

$$
u=A \log r+B,
$$

where $A$ and $B$ are constants. On the cylinders, which are equipotential surfaces, we have

$$
u_{a}=A \log a+B, \quad u_{b}=A \log b+B .
$$

The density $\sigma_{a}$ of the charge on the cylinder $r=a$ is

$$
\sigma_{a}=-\frac{\epsilon}{4 \pi}\left(\frac{\partial u}{\partial r}\right)_{r=a}=-\frac{\epsilon A}{4 \pi a},
$$

where $\epsilon$ denotes the dielectric constant of the field. The density $\sigma_{b}$ of the charge on the other cylinder will be

$$
\sigma_{b}=\frac{\epsilon}{4 \pi}\left(\frac{\partial u}{\partial r}\right)_{r=b}=\frac{\epsilon A}{4 \pi b} .
$$

Hence the charge on the cylinders, per unit length, will be $2 \pi a \sigma_{a}$ $=-\epsilon A / 2$ and $2 \pi b \sigma_{b}=\epsilon A / 2$, respectively. On the other hand the potential difference is

$$
u_{b}-u_{a}=A \log (b / a)
$$

The ratio

$$
\frac{\text { charge (per unit length) }}{\text { potential difference }}=\frac{\epsilon A / 2}{A \log (b / a)}=\frac{\epsilon}{2 \log (b / a)}
$$

An address delivered before the Pasadena meeting of the Society on November 25, 1944, by invitation of the Program Committee; received by the editors January 6 , 1945. 
is called the capacity (per unit length) of the jar. It measures the charge which is necessary in order to produce the potential difference 1.

(b) Another very elementary problem of electrostatics, related to the Leyden jar, is that of a spherical condenser. We consider two concentric, thin metallic spheres with radii $a$ and $b$, respectively, $a<b$. The potential $u$ of the field between these spheres depends only on the distance $r$ from the center; hence it must have the form

$$
u=A / r+B,
$$

where $A$ and $B$ are constants. On the spheres, which are equipotential surfaces, we have

$$
u_{a}=A / a+B, \quad u_{b}=A / b+B .
$$

The densities of the charges are in this case

$$
\sigma_{a}=-\frac{\epsilon}{4 \pi}\left(\frac{\partial u}{\partial r}\right)_{r=a}=\frac{\epsilon A}{4 \pi a^{2}}, \quad \sigma_{b}=\frac{\epsilon}{4 \pi}\left(\frac{\partial u}{\partial r}\right)_{r=b}=-\frac{\epsilon A}{4 \pi b^{2}},
$$

so that the total charges on the single spheres are $\pm \epsilon A$. The potential difference is

$$
u_{a}-u_{b}=A\left(a^{-1}-b^{-1}\right)
$$

The ratio

$$
\frac{\text { charge }}{\text { potential difference }}=\frac{\epsilon A}{A\left(a^{-1}-b^{-1}\right)}=\frac{\epsilon}{a^{-1}-b^{-1}}
$$

is called the capacity of the spherical condenser. In the limiting case when $b \rightarrow \infty$, we find for the capacity the expression $\epsilon a$. Hence assuming $\epsilon=1$, we find that the capacity of a sphere "with respect to an infinitely large sphere" is equal to the radius of the sphere. In general, the capacity has the dimension of a length.

2. Capacity of a condenser. (a) We consider two closed surfaces $s_{0}$ and $s_{1}$ such that $s_{1}$ is contained in the interior of $s_{0}$. We say that $s_{0}$ and $s_{1}$ form a "condenser" and we refer to the closed set of points exterior to $s_{1}$ and interior to $s_{0}$ as the "field." There is a point-function $u(p)$, harmonic in the field, such that $u(p)=0$ on $s_{0}$ and $u(p)=1$ on $s_{1}$. This function is uniquely determined. We call it the potential function of the condenser. It is the potential of certain electric charges, properly distributed on the surfaces $s_{0}$ and $s_{1}$.

The density of this charge is (assuming that the dielectric constant is 1 ) 


$$
-\frac{1}{4 \pi} \frac{\partial u}{\partial n}
$$

where $n$ is the normal directed into the interior of the field, that is, $n$ is the interior normal on $s_{0}$ and the exterior one on $s_{1}$. Hence the total charges will be

$$
-\frac{1}{4 \pi} \iint \frac{\partial u}{\partial n} d S \text { and }-\frac{1}{4 \pi} \iint \frac{\partial u}{\partial n} d S,
$$

respectively, the positive directions of the normals being determined as above.

Since $u$ is a harmonic function, these integrals are equal, except their sign. We form generally

$$
C=\frac{1}{4 \pi} \iint \frac{\partial u}{\partial n} d S
$$

where the integration is extended over an arbitrary closed surface lying in the interior of $s_{0}$ and containing $s_{1}$; the normal is the interior one. This integral is independent of the surface of the integration. It is a positive quantity, called the capacity of the condenser. The charges (2) on $s_{0}$ and $s_{1}$ are $-C$ and $+C$, respectively. The potential difference is in this case 1 so that this definition is a generalization of that given in 1 (b).

(b) According to the theory of harmonic functions the potential function $u(p)$ and the capacity $C$ of the condenser can also be obtained in the following way. We consider an arbitrary function $\psi(p)$ with continuous derivatives, defined in the field such that $\psi=0$ on $s_{0}$ and $\psi=1$ on $s_{1}$. We form Dirichlet's integral

$$
W(\psi)=\frac{1}{4 \pi} \iiint(\operatorname{grad} \psi)^{2} d V,
$$

where the integration is extended over the field. Then the minimum of $W(\psi)$ for the set of all functions mentioned is attained when $\psi(p)=u(p)$, and the minimum of $W(\psi)$ is equal to $C$. (See Kellogg $[8$, p. 279].1)

An alternate form of the integral $W(\psi)$ is

$$
W(\psi)=\iiint \psi \rho d V+\iint \psi \sigma d S,
$$

where

1 Numbers in brackets refer to the Bibliography at the end of this paper. 


$$
\rho=-\frac{\Delta \psi}{4 \pi}, \quad \sigma=-\frac{1}{4 \pi} \frac{\partial \psi}{\partial n}
$$

and the second integral in (5) is extended over $s_{0}+s_{1}$. The normal is directed into the interior of the field. The quantities (6) are the volume and surface density of a distribution of charges to which the potential $\psi$ corresponds. Here we assume that $\psi$ has continuous second derivatives. (See Kellogg [8, p. 278].) The expression $W(\psi) / 2$ can be interpreted as the potential energy of this distribution.

In the next sections we consider two important limiting cases of condensers.

3. Green's function of the exterior of a surface. (a) Let $s$ be a closed surface. Green's function $G(p)$ of the exterior of $s$ (with respect to the point of infinity) is characterized by the following properties:

1. $G(p)$ is a harmonic function at every finite point in the exterior of $s$.

2. If $r$ denotes the distance of $p$ from a fixed point, $\lim _{r \rightarrow \infty} r G(p)$ exists.

3. $G(p)=1$ if $p$ is on $s$.

The function $G$ is uniquely determined.

We recognize that this function is the limit of the potential function $u_{\rho}(p)$ of a condenser made up by $s_{1}=s$ and by a sphere $s_{0}$ with radius $\rho$ as $\rho \rightarrow \infty$. The field is in this case the exterior of $s$. The capacity $C$ of this condenser can be represented as in $\$ 2(3)$, that is,

$$
C=\frac{1}{4 \pi} \iint \frac{\partial G}{\partial n} d S,
$$

where the integration is extended over an arbitrary surface containing $s$.

In what follows, we refer to the function $G(p)$ and the constant $C$ as the potential function and the capacity of the surface $s$ (of the solid bounded by $s$, of the conductor bounded by $s$ ), respectively. The function $G(p)$ is the potential of certain electric charges on $s$ which are in electrostatic equilibrium because $s$ is, in view of property 3 , an equipotential surface. (Kellogg [8, p. 176].) The distribution of these charges is called the natural distribution. This is in general an inhomogeneous distribution. (In the case of a sphere it is homogeneous.)

The potential function just defined should not be confused with the potential of homogeneous charges on $s$. Also we make a clear distinction between the potential function and capacity of a condenser and of a surface (solid, conductor). 
(b) Introducing polar coordinates $r, \theta, \phi$ for the variable point $p$, we have for large $r$

$$
G(p)=\frac{Y_{0}(\theta, \phi)}{r}+\frac{Y_{1}(\theta, \phi)}{r^{2}}+\cdots+\frac{Y_{n}(\theta, \phi)}{r^{n+1}}+\cdots,
$$

where $Y_{n}(\theta, \phi)$ is a surface harmonic of order $n$. In view of the definition (1), we see that $Y_{0}(\theta, \phi)$ must be equal to $C$. Hence the capacity of a surface is the first coefficient in the expansion of the potential function $G(p)$ for large $r$.

An alternate definition of the potential function and capacity, similar to that in 2 (b), is possible.

Another characterization of the capacity and the natural distribution is the following (Riemann-Weber [28, p. 772]). We consider all positive charges on $s$ with the total charge 1 , defined by the density function $\mu(p), p$ on $s$ :

$$
\mu(p) \geqq 0, \quad \iint \mu(p) d S=1 .
$$

Then the integral

$$
\iiint \int \frac{\mu\left(p_{1}\right) \mu\left(p_{2}\right)}{r_{12}} d S_{1} d S_{2}
$$

where $p_{1}$ and $p_{2}$ run independently on $s$ ( $r_{12}$ is the distance of $p_{1}$ from $p_{2}, d S_{1}$ and $d S_{2}$ are the area elements at $p_{1}$ and $p_{2}$, respectively), will be a minimum if $\mu(p)$ is the density of the natural distribution. The minimum of this integral is $1 / C$.

For certain reasons which will become clear later, we call the capacity $C$ also the outer radius of the surface $s$ (of the solid bounded by $s$ ). In the case of a sphere it coincides with the ordinary radius.

4. Green's function of the interior of a surface. (a) Let $s$ be a closed surface, $q$ an arbitrary fixed point in the interior of $s$. Green's function $G(p ; q)$ of the interior of 6 with respect to $q$ is characterized by the following properties:

1. $G(p ; q)$ is a harmonic function in the interior of $s$ except at $q$.

2. If $r$ denotes the distance of $p$ from $q$, we have $G(p ; q)=1 / r$ $-H(p ; q)$, where $H(p ; q)$ is a harmonic function of $p$ throughout the interior of $s$.

3. $G=0$ if $p$ is on $s$.

The function $G$ is uniquely determined by $s$ and $q$.

We show that Green's function is closely related to the potential 
function of a properly defined condenser. We denote the value of $H$ at the point $p=q$ by $H_{0}$; since $H=1 / r$ on $s$, we see that $H_{0}>0$ and $H_{0}^{-1}$ is a mean-value of the distances $r$ of the points of $s$ from $q$.

Let $\epsilon$ be a positive number, $\epsilon H_{0}<1$. On the sphere

$$
r=\epsilon-H_{0} \epsilon^{2}
$$

we have

$$
G=1 /\left(\epsilon-H_{0} \epsilon^{2}\right)-H_{0}+O(\epsilon)=1 / \epsilon+O(\epsilon),
$$

where the term $O(\epsilon)$ divided by $\epsilon$ remains bounded as $\epsilon \rightarrow 0$. Let $u_{\epsilon}(p)$ be the potential function of the condenser formed by the sphere (1) and the surface $s$. Then the harmonic function

$$
u_{\epsilon}(p) / \epsilon-G(p ; q)
$$

is 0 on $s$ and equal to $1 / \epsilon-G=O(\epsilon)$ on the sphere (1). Hence by the maximum-minimum principle for harmonic functions we have

$$
u_{\epsilon}(p) / \epsilon-G(p ; q)=O(\epsilon)
$$

in the whole condenser. Consequently

$$
\lim _{\epsilon \rightarrow 0}\left(u_{\epsilon}(p) / \epsilon\right)=G(p ; q)
$$

at every point $p$ in the interior of $s$ different from $q$.

(b) We call the positive constant $H_{0}^{-1}$ the inner radius of $s$ with respect to the point $q$. As pointed out before, the inner radius is a certain mean-value of the distances of the points of $s$ from $q$. In the case of a sphere it coincides with the ordinary radius.

If we subject the surface $s$ to an inversion $\left(r^{\prime}=1 / r\right)$ with respect to $q$, it will be transformed into a surface $s^{\prime}$. It can be shown (cf. Kellogg $\left[8\right.$, p. 232]) that the exterior radius of $s^{\prime}$ is the reciprocal of the inner radius of $s$ with respect to $q$.

5. Generalization. The concept of the capacity or outer radius of a surface (of a solid bounded by a surface) can be generalized. Let us consider a finite number of mutually exclusive solids each bounded by a closed surface. The domain exterior to the set of these solids is connected. We can find again a function $G(p)$ harmonic in this domain for which $\lim _{r \rightarrow \infty} r G(p)$ exists and for which the boundary values are 1 on all the boundary surfaces. We call $G(p)$ the potential function of this set of solids (or of the set of surfaces). The capacity or outer radius of this set is defined as in $\$ 3$ (1) by taking as a surface of integration any closed surface enclosing the whole set in question. The alternate definitions can also be generalized. 
By a limiting process this definition can be further generalized and extended to an arbitrary closed set. The capacity or outer radius is an important monotonic set-function. Concerning its relation to certain problems of the Tchebychev type, cf. Pólya-Szegö [24].

Similar generalizations are possible for the inner radius.

6. Generalized condenser. Let us consider the mutually exclusive closed surfaces $s_{1}, s_{2}, \cdots, s_{n}$, and let the closed surface $s_{0}$ contain all these surfaces. The domain having the surfaces $s_{0}, s_{1}, \cdots, s_{n}$ as its boundary is called again the field.

We define a harmonic function $u_{i}(p)$ in the field assuming the boundary values

$$
u_{i}(p)=\left\{\begin{array}{l}
1 \text { on } s_{i}, \\
0 \text { on } s_{k}, k \neq i ; \quad i, k=0,1, \cdots, n .
\end{array}\right.
$$

We introduce the integrals

$$
C_{i k}=-\frac{1}{4 \pi} \iint_{s_{k}} \frac{\partial u_{i}}{\partial n} d S,
$$

where the integration is extended over the surface $s_{k}$ and the normal is directed into the interior of the field. Obviously $C_{i k}$ is positive if $i=k$ and is negative if $i \neq k$. We call the coefficients $C_{i k}$ the capacity coefficients.

By Green's identity we conclude

$$
C_{i 0}+C_{i 1}+\cdots+C_{i n}=0, \quad i=0,1, \cdots, n .
$$

Also

$$
u_{0}(p)+u_{1}(p)+\cdots+u_{n}(p)=1,
$$

since the function on the left-hand side of (4) has the boundary value 1 on all surfaces $s_{i}$.

By Green's identity we find further

$$
\begin{aligned}
& \frac{1}{4 \pi} \iiint \operatorname{grad} u_{i} \cdot \operatorname{grad} u_{k} d V \\
& =-\frac{1}{4 \pi} \iint u_{k} \frac{\partial u_{i}}{\partial n} d S-\frac{1}{4 \pi} \iiint u_{k} \Delta u_{i} d V
\end{aligned}
$$

The volume integrals are extended over the field, the surface integrals over the complete boundary. Since $u_{k}=0$ on all the surfaces except on $s_{k}$, and $\Delta u_{i}=0$, we find 


$$
\frac{1}{4 \pi} \iiint \operatorname{grad} u_{i} \cdot \operatorname{grad} u_{k} d V=C_{i k}
$$

This new representation implies the symmetry relation

$$
C_{i k}=C_{k i} \text {. }
$$

Another remarkable corollary of (6) is the following identity involving the arbitrary real numbers $\alpha_{0}, \alpha_{1}, \cdots, \alpha_{n}$ :

$$
\sum_{i=0}^{n} \sum_{k=0}^{n} C_{i k} \alpha_{i} \alpha_{k}=\frac{1}{4 \pi} \iiint\left(\sum_{i=0}^{n} \alpha_{i} \operatorname{grad} u_{i}\right)^{2} d V .
$$

Thus the quadratic form on the left-hand side of (8) is non-negative. It is easy to show that the form arising if $i$ and $k$ run from 1 to $n$ is positive.

If we write

$$
u(p)=\alpha_{0} u_{0}(p)+\alpha_{1} u_{1}(p)+\cdots+\alpha_{n} u_{n}(p),
$$

the function $u(p)$ will be harmonic in the field and $u=\alpha_{k}$ on $s_{k}$; that is, $\alpha_{k}$ is the constant value of the potential $u$ on the conductor $s_{k}$. The "charge" of $u$ on $s_{k}$ is

$$
\beta_{k}=-\frac{1}{4 \pi} \iint_{s_{k}} \frac{\partial u}{\partial n} d S=\alpha_{0} C_{0 k}+\alpha_{1} C_{1 k}+\cdots+\alpha_{n} C_{n k}
$$

Obviously

$$
\beta_{0}+\beta_{1}+\cdots+\beta_{n}=0 .
$$

If the charges $\beta_{k}$ are prescribed such that they satisfy the relation (11), the system (10) of linear equations can be solved for the $\alpha_{k}$. Doing this, we can disregard the equation corresponding to $k=0$ because it is a consequence of the others. They can be written as follows:

$$
\begin{aligned}
\left(\alpha_{1}-\alpha_{0}\right) C_{1 k}+\left(\alpha_{2}-\alpha_{0}\right) C_{2 k}+\cdots+\left(\alpha_{n}-\alpha_{0}\right) C_{n k} & =\beta_{k}, \\
k & =1,2, \cdots, n .
\end{aligned}
$$

The determinant of this system is different from zero and so

$$
\alpha_{i}-\alpha_{0}=D_{i 1} \beta_{1}+D_{i 2} \beta_{2}+\cdots+D_{i n} \beta_{n}, \quad i=1,2, \cdots, n,
$$

where the constants $D_{i k}$ are uniquely determined. They are called the potential coefficients. Physical considerations show that they are all positive. The mathematical background of this fact is elucidated by a theorem of Stieltjes [30, p. 82].

The simplest non-trivial example of a generalized condenser is the 
case $n=2$ when $s_{1}$ and $s_{2}$ are two mutually exclusive spheres and $s_{0}$ is a sphere with infinitely large radius. (Cf. \$16.)

7. Cylindrical (two-dimensional) problems. (a) As a generalization of the Leyden jar we consider two infinite cylinders with parallel generators, one containing the other. We intersect these cylinders perpendicularly to the generators and denote by $l_{0}$ the exterior and by $l_{1}$ the interior contour of the cross section. Let $u$ denote the harmonic function in the field between the two cylinders which assumes the constant value 0 on the exterior and the constant value 1 on the interior cylinder. This function is constant along any line in the field parallel to the generators. It is a two-dimensional harmonic function.

The density of the charge on the cylinders is

$$
-\frac{\epsilon}{4 \pi} \frac{\partial u}{\partial n},
$$

where $\epsilon$ is the dielectric constant and $n$ denotes the normal directed into the field. The total charge on the cylinders, per unit length, will be

$$
-\frac{\epsilon}{4 \pi} \int \frac{\partial u}{\partial n} d L,
$$

where the integration is extended along $l_{0}$ and $l_{1}$, respectively, and the normal is defined as before.

(b) We assume $\epsilon=1$ and from now on we consider the problem in the plane. Let $l_{0}$ and $l_{1}$ be two closed curves, $l_{1}$ in the interior of $l_{0}$; we say that $l_{0}$ and $l_{1}$ form a "two-dimensional condenser." The closed set between the curves is called the field. The potential function $u(p)$ of this condenser is a two-dimensional harmonic function in the field, satisfying the relations $u(p)=0$ on $l_{0}$ and $u(p)=1$ on $l_{1}$. The "twodimensional capacity" $c$ of this condenser is defined by

$$
c=\frac{1}{2 \pi} \int \frac{\partial u}{\partial n} d L,
$$

where the integration is extended over an arbitrary closed curve lying in the interior of $l_{0}$ and containing $l_{1}$; the normal is the interior one. Since $u$ is a harmonic function, this integral is independent of the line of integration. The capacity $c$ is always positive. The charges (2) are $\pm c / 2$. If $c$ is the "two-dimensional capacity" of the two-dimensional condenser defined above, the capacity (per unit length) of the cylindrical condenser in space will be $c / 2$. In case of the Leyden jar, dis- 
cussed in $\$ 1$, we have $(\epsilon=1)$

$$
u=(\log b-\log r) /(\log b-\log a), \quad c=1 / \log (b / a) .
$$

As in space, an alternate definition is possible by means of Dirichlet's integral.

(c) Let $l$ be a closed curve in the plane. Green's function $G(p)$ of the exterior of $l$ (with respect to the point of infinity) is characterized by the following properties:

1. $G(p)$ is a harmonic function at every finite point in the exterior of $l$.

2. If $r$ denotes the distance of $p$ from a fixed point, we have for large $r$

$$
\begin{gathered}
G(p)=\log \frac{1}{r}-\log \frac{1}{\bar{r}}+\frac{a_{1} \cos \phi+b_{1} \sin \phi}{r} \\
+\frac{a_{2} \cos 2 \phi+b_{2} \sin 2 \phi}{r^{2}}+\cdots,
\end{gathered}
$$

where $\bar{r}$ is a certain positive constant and $a_{1}, b_{1}, a_{2}, b_{2}, \cdots$ are real constants.

3. $G(p)=0$ if $p$ is on $l$.

The function $G(p)$ is uniquely determined.

We refer to the constant $\bar{r}$ as the outer radius or transfinite diameter of $l$ (of the domain bounded by $l$ ). ${ }^{2}$

Let us consider the two-dimensional condenser made up by $l_{1}=l$ and by a circle $l_{0}$ of radius $\rho$. Denoting its potential by $u_{\rho}(p)$ and capacity by $c_{\rho}$, we can show by an argument similar to that in $\$ 4$ that

$$
\begin{aligned}
\left(u_{\rho}(p)-1\right) \log (\rho / \bar{r})-G(p) & =O(1 / \rho), \\
\lim _{\rho \rightarrow \infty}\left(u_{\rho}(p)-1\right) \log \rho & =G(p),
\end{aligned}
$$

provided $p$ is in the exterior of $l$. Moreover

$$
c_{\rho} \log (\rho / \bar{r})-1=O(1 / \rho), \quad \lim _{\rho \rightarrow \infty} c_{\rho} \log \rho=1 .
$$

The definition of the outer radius can be extended to an arbitrary closed set. It is a monotonic set-function. Concerning its relation to certain problems of the Tchebychev type, cf. Pólya-Szegö [24].

In order to avoid confusion, we point out that the term outer radius of a plane set can be taken in the "two-dimensional" or "three-dimen-

2 In Pólya-Szegö [24] this constant was designated also as the capacity constant. In the present exposition we prefer not to use this term. 
sional" sense. For instance, the outer radius of a circular disk of radius 1 is equal to 1 in the first sense and to $2 / \pi$ in the second sense.

(d) Let $l$ be again a closed curve in the plane, $q$ an arbitrary fixed point in the interior of $l$. Green's function $G(p ; q)$ of the interior of $l$ with respect to $q$ is characterized by the following properties:

1. $G(p ; q)$ is a harmonic function in the interior of $l$ except for $p=q$.

2. If $r$ denotes the distance of $p$ from $q$, we have

$$
G(p ; q)=\log (1 / r)-H(p ; q),
$$

where $H(p ; q)$ is a harmonic function of $p$ throughout the interior of $l$. 3. $G=0$ if $p$ is on $l$.

The function $G$ is uniquely determined by $l$ and $q$.

This concept is again closely related to the potential of a properly defined condenser. We denote the value of $H$ at the point $p=q$ by $H_{0}$ and write $H_{0}=\log \left(1 / r_{q}\right)$. Then the positive constant $r_{q}$ is called the inner radius of $l$ with respect to $q$. Since $H=\log (1 / r)$ on $l$, we see that $H_{0}=\log \left(1 / r_{q}\right)$ must be a mean-value of the values $\log (1 / r)$, hence $r_{q}$ must be a mean-value of the distances $r$ of the points of $l$ from $q$.

8. Conformal mapping. The concepts of outer and inner radii in the plane are closely related to the conformal mapping.

(a) Let $l$ be a closed curve in the plane. The exterior of $l$ can be mapped conformally onto the exterior of a circle such that the point at infinity and the line element (that is, the magnitude and direction of the line-element) at infinity are preserved. The radius of this circle is uniquely determined by $l$. It is identical with the outer radius $\bar{r}$ of $l$.

If we introduce complex numbers $z$ in the plane of $l$ and complex numbers $w$ in the plane of the circle, the mapping can be represented in the form $w=f(z)$ where $f(z)$ is regular in the finite $z$-plane outside of $l$ and has a simple pole at $z=\infty$. It maps the exterior of $l$ onto the domain $|w|>\bar{r}$. For large $|z|$ we have

$$
w=f(z)=z+c_{0}+c_{-1} z^{-1}+c_{-2} z^{-2}+\cdots,
$$

where the coefficients $c_{0}, c_{-1}, \cdots$ are uniquely determined complex numbers.

Green's function $G(p)$ can be represented in terms of the map function $f(z)$. If the point $p$ corresponds to the complex number $z$, we have

$$
G(p)=\log (\bar{r} /|f(z)|) .
$$

(b) Let $l$ again be a closed curve, and $q$ a fixed point in the interior of $l$. The interior of $l$ can be mapped conformally onto the interior of a circle with center at $q$ such that the point $q$ and the line-element at $q$ 
are preserved. The radius of this circle is uniquely determined by $l$ and $q$. It is identical with the inner radius $r_{q}$ of $l$ with respect to $q$.

If we introduce complex numbers $z$ in the plane of $l$ and complex numbers $w$ in the plane of the circle, and if $q$ is the point $z=w=0$, this mapping can be represented in the form $w=g(z)$, where $g(z)$ is regular in the interior of $l, g(0)=0, g^{\prime}(0)=1$. It maps the interior of $l$ onto the circle $|w|<r_{q}$. For sufficiently small $|z|$ we have

$$
w=g(z)=z+c_{2} z^{2}+c_{3} z^{3}+\cdots,
$$

where the coefficients $c_{2}, c_{3}, \cdots$ are uniquely determined complex numbers.

Green's function $G(p ; q)$ can be represented in terms of this map function $g(z)$. If $p$ is the point $z$, we have

$$
G(p ; q)=\log \left(r_{q} /|g(z)|\right) \text {. }
$$

9. Symmetrization in space. (a) The capacity or outer radius $C=C(S)$ of a solid $S$ is an interesting function which can be compared with other important set-functions, like the volume $V(S)$, the area $A(S)$ or the diameter $D(S)$. In case $S$ is convex, the integral $M(S)$ of the mean curvature can also be considered. It is obvious that $C(S)$ is "monotonic" and that in case of a sphere $C(S)$ is equal to the radius of this sphere.

In the theory of convex sets various inequalities are established between the quantities mentioned $[2, \mathrm{p} .84]$. These results are centered around the classical isoperimetric inequality:

$$
A^{3}-36 \pi V^{2} \geqq 0,
$$

with equality only for the sphere. This inequality holds also for nonconvex solids. Thus: Of all solids with given area, the sphere has the maximum volume.

Another important inequality, holding for convex solids, is the following:

$$
M^{2}-4 \pi A \geqq 0,
$$

with equality only for the sphere. Thus: Of all convex solids with given area, the sphere has the minimum integral of the mean curvature.

Another way of expressing these inequalities is the following. Let $R_{V}=[3 V /(4 \pi)]^{1 / 3}$ be the radius of the sphere with a volume equal to that of the given solid $S$. Let $R_{A}=[A /(4 \pi)]^{1 / 2}$ and $R_{M}=M /(4 \pi)$ have the analogous meaning taking the area and the integral of the mean curvature instead of the volume. Then 


$$
R_{M} \geqq R_{A} \geqq R_{V} .
$$

In the first inequality $S$ must be convex, in the second $S$ may be arbitrary.

(b) In the study of the isoperimetric inequality (1) and in related problems the idea of symmetrization is fundamental.

Let us consider a plane $\epsilon$, a straight line $e$, and a point $E$. The symmetrization of the solid $S$ with respect to the plane $\epsilon$ is defined as a geometric transformation changing the solid $S$ into a solid $S^{\prime}$ symmetric with respect to $\epsilon$ and characterized by the following properties. Any straight line perpendicular to $\epsilon$ and intersecting one of the solids $S$ and $S^{\prime}$ intersects also the other and both intersections have the same length. The intersection with $S$ may consist of several segments whose total length has to be taken; the intersection with $S^{\prime}$ is either a segment or a point.

Symmetrization with respect to the line $e$ changes $S$ into a solid of revolution $S^{\prime \prime}$ with axis $e$, defined by the following property. Any plane perpendicular to $e$ and intersecting one of the solids $S$ and $S^{\prime \prime}$ intersects also the other and both intersections have the same area; the intersection with $S^{\prime \prime \prime}$ is either a circular disk or a point.

Symmetrization with respect to the point $E$ changes the solid $S$ into a sphere $S^{\prime \prime \prime}$ with center at $E ; S$ and $S^{\prime \prime \prime}$ have the same volume.

The first kind of symmetrization, repeated for a suitably chosen sequence of planes, generates the other two kinds of symmetrization.

All three kinds of symmetrization preserve the volume and diminish (or leave unchanged) the area of the solid. This is a fundamental result; see, for instance, Bonnesen-Fenchel [2, pp. 69-72]. That part of the result which is concerned with the symmetrization with respect to a point is the classical isoperimetric property of the sphere expressed by the inequality (1).

(c) Let us consider a condenser made up by the closed surfaces $s_{0}$ and $s_{1}$. We symmetrize the condenser by symmetrizing the two solids bounded by $s_{0}$ and $s_{1}$ simultaneously, with respect to the same plane or line or point.

All three kinds of symmetrization diminish (or leave unchanged) the capacity of the condenser. That part of this statement which is concerned with the symmetrization with respect to a point was discovered by Poincaré [20, pp. 17-22]. A different proof was suggested by Faber [4] and the first complete proof was given by Szegö [32]. The two other parts are due to Pólya-Szegö [25]. Poincaré's theorem can be expressed in the form 
From the previous general result, by proper limiting processes, we conclude [25]:

All three kinds of symmetrization decrease (or leave unchanged) the outer radius (capacity) of a surface; they increase (or leave unchanged) the inner radius of a surface with respect to an interior point. ${ }^{3}$

As a corollary we note:

The inner radius of a surface with respect to an interior point is never greater than the outer radius.

Another interesting corollary is the following [25]:

The outer radius of a cylindrical solid is never less than the outer radius of a right circular cylinder of the same height whose base has the same area as that of the given solid.

An analogous extremal property holds for the right circular cone.

A limiting case of the above statement arises when the height of the cylinder becomes 0 . Since the outer radius of a circular disk of radius $a$ is $2 a / \pi(\$ 12(11))$, we have:

The outer radius of a disk (in the three-dimensional sense) is never less than $2 / \pi$ times the radius of a circle which has the same area as the given disk.

10. Symmetrization in the plane. The analogous considerations in the plane are rather obvious.

In the plane we have two kinds of symmetrization, one with respect to a straight line $e$ and another with respect to a point $E$.

Let $l$ be a closed curve in the plane and $A$ the area enclosed by $l$, $L$ the length of $l, \bar{r}$ the outer radius of $l$ (in the two-dimensional sense), $r_{q}$ the inner radius of $l$ with respect to a point $q$ in the interior of $l, C$ the outer radius (capacity) of the disk bounded by $l$ (in the three-dimensional sense). Then we have:

Both kinds of symmetrization increase (or leave unchanged) $r_{q},{ }^{4}$ preserve $A$ and diminish (or leave unchanged) $L, \bar{r}$, and $C$.

Let us consider first the symmetrization with respect to a line. The part of the above statement concerning $A$ is trivial, and that concerning $L$ is classical (Bonnesen-Fenchel $[2$, p. 72]). The other parts are due to Pólya-Szegö [25].

${ }^{3}$ In this case the plane of symmetrization must go through the point $q$ (similarly for the symmetrization with respect to a line or a point).

- See footnote 3 . 
As to the symmetrization with respect to a point, the statement about $A$ is trivial and that concerning $L$ is equivalent to the isoperimetric property of the circle. The statements about $r_{q}$ and $\bar{r}$ are equivalent to the area theorems of Bieberbach (see Pólya-Szegö [23, vol. 2 , p. 21 , problem 125 , p. 22 , problem 126]). Concerning extensions of these statements to multiply connected domains, see Pólya [22] and Szegö [31]. The part concerning $C$ is due to Pólya-Szegö [25]. (Cf. the last theorem in \$9.)

We note the following inequalities between the quantities mentioned:

$$
r_{q} \leqq\left(\frac{A}{\pi}\right)^{1 / 2} \leqq \frac{\pi}{2} C, \quad\left(\frac{A}{\pi}\right)^{1 / 2} \leqq \bar{r} \leqq \frac{L}{2 \pi} .
$$

They all follow from the above statement except the last one which is of a different nature (see, for instance, Pólya-Szegö [23, vol. 2, p. 21, problem 124]). As a special corollary we mention:

$$
r_{q} \leqq \bar{r}
$$

11. Parallel surfaces. (a) By the diameter $D$ of a surface we mean the greatest distance between any two of its points.

Considering all closed surfaces $s$ with given diameter, the capacity $C$ of $s$ is a maximum if and only if $s$ is a sphere.

Concerning this extremum property of the sphere, cf. Maxwell [11, p. 117] and Szegö [33]. It can be expressed in the form of an inequality:

$$
D \geqq 2 C \text {. }
$$

The analogous inequality for the inner radius follows from here since this radius is never greater than $C$ (cf. \$9).

It suffices to prove (1) for convex surfaces. The proof given by Szegö is based on the use of parallel surfaces. If we denote by $s_{h}$ the surface parallel to $s$ in the distance $h$ and by $C(h)$ the capacity of $s_{h}$, the derivative $C^{\prime}(h)$ can be computed. From this not only (1) but the following more precise inequality follows:

Of all closed convex surfaces $s$ with given integral $M$ of the mean curvature, the capacity of $s$ is a maximum if and only if $s$ is a sphere.

This is equivalent to the inequality

$$
M \geqq 4 \pi C,
$$

which is indeed more precise than (1) since $2 \pi D \geqq M$. 
Recapitulating, we found the following inequalities for $C, V, D, M$ :

$$
D / 2 \geqq R_{M} \geqq C \geqq R_{V} .
$$

(b) The following inequalities for the capacity of a condenser are due to Pólya-Szegö [25].

Let the inner surface $s_{1}$ of a condenser of capacity $C$ be convex and the outer surface $s_{0}$ be parallel to $s_{1}$. Let $h$ be the distance between $s_{0}$ and $s_{1}$, $A$ the area of the surface $s_{1}$ and $M$ the integral of the mean curvature of $s_{1}$. Then

$$
A /(4 \pi h)<C<A /(4 \pi h)+M /(4 \pi) .
$$

For concentric spheres the second inequality becomes an equation.

The lower bound is a customary approximation for $C$ in case $h$ is small; see for instance Maxwell [11, p. 150]. The upper bound is suggested by certain approximations due to Clausius [3, p. 43]. Concerning the limiting case $h \rightarrow \infty$, see (2). An improvement of the upper bound is the following inequality (Pólya-Szegö [25]):

$$
\begin{aligned}
\frac{1}{C} & \geqq \frac{2 \pi}{\lambda M} \log \left(\frac{(1-\lambda) M+4 \pi h}{(1+\lambda) M+4 \pi h} \cdot \frac{1+\lambda}{1-\lambda}\right), \\
\lambda & =\left(1-4 \pi A M^{-2}\right)^{1 / 2} .
\end{aligned}
$$

(c) Finally we point out the inequalities corresponding to (4) in the two-dimensional case.

Let $l_{1}$ be a closed convex curve in the plane and $l_{0}$ an arbitrary outer curve parallel to $l_{1}$. We denote by $c$ the capacity of the two-dimensional condenser bounded by $l_{0}$ and $l_{1}$. If $L_{0}$ is the length of $l_{0}$ and $L_{1}$ the length of $l_{1}$, we have

$$
\left(L_{0} / L_{1}-1\right)^{-1}<c<\left\{\log \left(L_{0} / L_{1}\right)\right\}^{-1}
$$

unless $l_{1}$ is a circle.

12. Example 1. Ellipsoid. (a) Let $a, b, c$ be three constants, $a>b>c>0$. We consider the quadrics

$$
\frac{x^{2}}{a^{2}+\rho}+\frac{y^{2}}{b^{2}+\rho}+\frac{z^{2}}{c^{2}+\rho}=1,
$$

where $\rho$ is a real parameter. For any point $(x, y, z)$ not in a coordinate plane, this equation has three distinct real roots in $\rho$ which we denote by $\lambda, \mu, \nu$ : 


$$
-a^{2}<\nu<-b^{2}<\mu<-c^{2}<\lambda .
$$

They are called the ellipsoidal coordinates. (See, for instance, Kellogg $[8$, p. 186].) On the ellipsoid

$$
x^{2} / a^{2}+y^{2} / b^{2}+z^{2} / c^{2}=1
$$

we have $\lambda=0$. By means of these coordinates various condenser and conductor problems related to ellipsoids can be discussed in a very elegant way. We consider the problem of electrostatic equilibrium on the ellipsoid (3) (see, for instance, Kellogg [8, pp. 188-191]). If the charge on the ellipsoid is 1 , the potential at an outside point $p(x, y, z)$ will be

$$
u(p)=\frac{1}{2} \int_{\lambda}^{\infty} \frac{d t}{\left\{\left(a^{2}+t\right)\left(b^{2}+t\right)\left(c^{2}+t\right)\right\}^{1 / 2}},
$$

where $\lambda$ has the meaning above, $\lambda \geqq 0$. For the capacity $C$ we find

$$
\frac{1}{C}=\frac{1}{2} \int_{0}^{\infty} \frac{d t}{\left\{\left(a^{2}+t\right)\left(b^{2}+t\right)\left(c^{2}+t\right)\right\}^{1 / 2}} .
$$

The density of the charge on the ellipsoid is

$$
\sigma=d / 4 \pi a b c,
$$

where $d$ is the distance from the center of the tangent plane at the point $(x, y, z)$.

The problem of a condenser bounded by two confocal ellipsoids can also be treated.

(b) We denote by $\alpha, \beta, \gamma$ the numerical eccentricities of the principal sections of the ellipsoid (3):

$$
\alpha^{2}=\frac{b^{2}-c^{2}}{b^{2}}, \quad \beta^{2}=\frac{a^{2}-c^{2}}{a^{2}}, \quad \gamma^{2}=\frac{a^{2}-b^{2}}{a^{2}} .
$$

Then we have the following remarkable representations (Pólyz-Szegö [25]):

$$
\begin{aligned}
\frac{1}{C} & =\frac{1}{a} \sum_{n=0}^{\infty} \frac{(\beta \gamma)^{n}}{2 n+1} P_{n}\left(\frac{\beta^{2}+\gamma^{2}}{2 \beta \gamma}\right) \\
& =\frac{c}{a b} \sum_{n=0}^{\infty} \frac{2 \cdot 4 \cdots 2 n}{1 \cdot 3 \cdots(2 n+1)}(\alpha \beta)^{n} P_{n}\left(\frac{\alpha^{2}+\beta^{2}}{2 \alpha \beta}\right),
\end{aligned}
$$

where $P_{n}(x)$ denotes Legendre's polynomial.

In the special case of a prolate ellipsoid we have $b=c, \alpha=0, \beta=\gamma$, and 


$$
C^{-1}=(2 a \beta)^{-1} \log \frac{1+\beta}{1-\beta} .
$$

In the special case of an oblate ellipsoid we have $a=b, \alpha=\beta, \gamma=0$, and

$$
C^{-1}=(a \beta)^{-1} \operatorname{arc} \sin \beta .
$$

Another interesting special case is that of an elliptical disk. Then $c=0$ and we have

$$
\frac{1}{C}=\int_{0}^{\pi / 2} \frac{d \phi}{\left(a^{2} \cos ^{2} \phi+b^{2} \sin ^{2} \phi\right)^{1 / 2}},
$$

that is, $C$ is the arithmetic-geometric mean of the semi-axes $a, b$ multiplied by $2 / \pi$. In the case of a circular disk, $a=b$, we find $C=2 a / \pi$.

(c) In the special case of the ellipsoid (3), the inequalities (3) of $\$ 11$ have been refined by Pólya-Szegö [25] (cf. also Pólya-Szegö [21], Watson [36], Szegö [34]) in the following way:

$$
\begin{aligned}
R_{V}=(a b c)^{1 / 3} & <\frac{(b c)^{1 / 2}+(c a)^{1 / 2}+(a b)^{1 / 2}}{3}<C \\
& <\frac{a+b+c}{3}<R_{M}<a .
\end{aligned}
$$

As to the radius $R_{A}$, it turns out that $C$ and $R_{A}$ are in general not "comparable." But we have (Pólya-Szegö [25])

$$
C \gtrless R_{A}
$$

for an ellipsoid of revolution, according as it is prolate or oblate. It is interesting to observe that for such an ellipsoid

$$
C / R_{A}=1 \pm \frac{2}{945} \beta^{6}+\cdots
$$

where the signs \pm correspond to prolate and oblate ellipsoids, respectively. The neglected terms contain higher powers of $\beta$. (See Aichi [1], Lord Rayleigh [26] and Russell [29].)

From (11) we conclude that the capacity of an elliptical disk with semi-axes $a, b$ does not change if we replace $a, b$ by $(a+b) / 2$ and $(a b)^{1 / 2}$ (cf. Kellogg [8, p. 61]). In an analogous way, Pólya-Szegö [25] investigated certain transformations, related to the Landen transformation of elliptic integrals, which carry ellipsoids into ellipsoids preserving the capacity. 
13. Peripolar (toroidal) and dipolar coordinates. (a) Let $a>0$. In the complex $\sigma+i t$ plane we introduce the coordinates $\eta, \theta$ of a point $p(\sigma, t)$ by the following equation:

$$
\sigma+i t=a \frac{1+e^{-\eta+i \theta}}{1-e^{-\eta+i \theta}}
$$

or

$$
\sigma=a(\cosh \eta-\cos \theta)^{-1} \sinh \eta, \quad t=a(\cosh \eta-\cos \theta)^{-1} \sin \theta .
$$

The geometric meanings of $\eta$ and $\theta$ are obvious. Let $A$ and $B$ be the two points $-a$ and $+a$ on the real $\sigma$-axis; then

$$
\eta=\log (A p / B p), \quad \theta=\operatorname{angle}(A p B) .
$$

Of course $\theta$ is determined only mod $2 \pi$. For the points in the upper half-plane $(t>0)$ we take $0<\theta<\pi, \bmod 2 \pi$, and for the points in the lower half-plane $(t<0)$ we take $\pi<\theta<2 \pi, \bmod 2 \pi$. For the point at infinity we have $\theta=0, \bmod 2 \pi$, on the line $A B$ we have $\theta=\pi, \bmod 2 \pi$, and on the part of the real $\sigma$-axis outside $A B$ we have $\theta=0, \bmod 2 \pi$.

(b) Let us consider a rectangular coordinate system in space and a point $p$ with coordinates $x, y, z$. We define the peripolar (toroidal) coordinates $\eta, \theta, \phi$ of $p$ in the following way (Riemann [27], C. Neumann [15], Mehler [12]). We put a plane through $p$ and the $z$-axis which forms the azimuthal angle $\phi$ with the $x z$-plane. (This plane is undetermined when $p$ is on the $z$-axis.) In this plane we introduce the coordinates $\sigma, t ; \eta, \theta$ as before, writing $x=\sigma \cos \phi, y=\sigma \sin \phi, z=t$; thus

$$
\begin{aligned}
& x=a(\cosh \eta-\cos \theta)^{-1} \sinh \eta \cos \phi, \\
& y=a(\cosh \eta-\cos \theta)^{-1} \sinh \eta \sin \phi, \\
& z=a(\cosh \eta-\cos \theta)^{-1} \sin \theta .
\end{aligned}
$$

The range of these coordinates is $\eta \geqq 0,0 \leqq \theta<2 \pi, 0 \leqq \phi<2 \pi$.

(c) We define the dipolar coordinates (C. Neumann [18]) of the point $p$ in a similar manner by putting a plane through $p$ and the $x$-axis which forms the azimuthal angle $\phi$ with the $x y$-plane. In this plane we introduce $\sigma, t ; \eta, \theta$ as before, writing $x=\sigma, y=t \cos \phi$, $z=t \sin \phi ;$ we find in this case

$$
\begin{aligned}
& x=a(\cosh \eta-\cos \theta)^{-1} \sinh \eta, \\
& y=a(\cosh \eta-\cos \theta)^{-1} \sin \theta \cos \phi, \\
& z=a(\cosh \eta-\cos \theta)^{-1} \sin \theta \sin \phi .
\end{aligned}
$$

The range of these co-ordinates is $-\infty<\eta<\infty, \dot{0}<\theta \leqq \pi, 0 \leqq \phi<2 \pi$. 
We apply the same symbols $\eta, \theta, \phi$ for both kinds of coordinates; no confusion is possible since in each definite problem only one kind of coordinates is used.

14. Example 2. Anchor ring (torus). In peripolar coordinates an anchor ring is defined by the condition $\eta=\eta_{0}>0$. In the exterior space we have $0<\eta<\eta_{0}$. The potential function of the ring is (Hicks [6]):

(1) $u(p)=\frac{2^{3 / 2}}{\pi}(\cosh \eta-\cos \theta)^{1 / 2} \sum_{n=0}^{\infty} \frac{Q_{n-1 / 2}\left(\cosh \eta_{0}\right)}{P_{n-1 / 2}\left(\cosh \eta_{0}\right)} \cos n \theta P_{n-1 / 2}(\cosh \eta)$.

The symbol $\sum^{\prime}$ indicates that the term $n=0$ has to be multiplied by $1 / 2$. The functions ${ }^{5}$

$$
\begin{aligned}
& P_{n-1 / 2}(\cosh \eta)=e^{-(n+1 / 2) \eta} F\left(1 / 2, n+1 / 2 ; 1 ; 1-e^{-2 \eta}\right), \\
& Q_{n-1 / 2}(\cosh \eta) \\
& \quad=\pi \frac{1 \cdot 3 \cdots(2 n-1)}{2 \cdot 4 \cdots 2 n} e^{-(n+1 / 2) \eta} F\left(1 / 2, n+1 / 2 ; n+1 ; e^{-2 \eta}\right),
\end{aligned}
$$

where $F$ is the hypergeometric function of Gauss and $\eta>0$, satisfy the differential equation

$$
d^{2} y / d \eta^{2}+\operatorname{coth} \eta d y / d \eta+\left(1 / 4-n^{2}\right) y=0 .
$$

This differential equation arises from Legendre's equation (satisfied by the Legendre functions $P_{n}(\cosh \eta)$ and $\left.Q_{n}(\cosh \eta)\right)$ by replacing $n$ by $n-1 / 2$. The functions

$$
(\cosh \eta-\cos \theta)^{1 / 2} \cos n \theta\left\{\begin{array}{l}
P_{n-1 / 2}(\cosh \eta) \\
Q_{n-1 / 2}(\cosh \eta)
\end{array}\right.
$$

are harmonic. They are called the ring (toroidal) functions. As to the convergence of (1) we note that $Q_{n-1 / 2}(\cosh \eta)$ and $P_{n-1 / 2}(\cosh \eta)$ are of the order $e^{-n \eta}$ and $e^{n \eta}$, respectively, as $n \rightarrow \infty$.

The capacity of the anchor ring is

$$
C=\frac{4 a}{\pi} \sum_{n=0}^{\infty} \frac{Q_{n-1 / 2}\left(\cosh \eta_{0}\right)}{P_{n-1 / 2}\left(\cosh \eta_{0}\right)} .
$$

Taking the two first terms of this sum, we have the following approximation of the capacity [6, p. 643]:

$$
2 a K / K^{\prime}+4 a(K-E) / E^{\prime},
$$

where $K$ and $E$ denote, as usual, the complete elliptic integrals of the first and second kind, and the modulus is

\footnotetext{
5 The constant factor in $Q_{n-1 / 2}$ has to be replaced by 1 for $n=0$.
} 


$$
k=e^{-\eta_{0}}=R^{-1}\left(D-\left(D^{2}-R^{2}\right)^{1 / 2}\right) .
$$

Here $D$ is the distance of the center of the revolving disk, which generates the ring, from the $z$-axis and $R$ is the radius of the disk.

Other problems connected with anchor rings have been treated by Niven [19].

15. Example 3. Spindle. In dipolar coordinates a spindle is defined by the condition $\theta=\theta_{0}, 0<\theta_{0}<\pi$. In the exterior space we have $0<\theta<\theta_{0}$. The potential function of the spindle is (C. Neumann [18, p. 224])

(1) $u(p)=2^{-1 / 2}(\cosh \eta-\cos \theta)^{1 / 2} \int_{-\infty}^{\infty} \frac{L_{q}\left(\cos \theta_{0}\right) L_{q}(-\cos \theta)}{L_{q}\left(-\cos \theta_{0}\right)} \frac{\cos q \eta}{\cosh q \pi} d q$.

The function

$$
\begin{aligned}
L_{q}(\cos \theta) & =\pi^{-1} \cosh q \pi \int_{-\infty}^{\infty}\left(2 \cosh \eta^{\prime}-2 \cos \theta\right)^{-1 / 2} \cos q \eta^{\prime} d \eta^{\prime} \\
& =F\left(1 / 2+i q, 1 / 2-i q ; 1 ; \cos ^{2}(\theta / 2)\right)
\end{aligned}
$$

satisfies the differential equation

$$
d^{2} y / d \theta^{2}+\cot \theta d y / d \theta-\left(q^{2}+1 / 4\right) y=0 .
$$

This equation arises from Legendre's differential equation (satisfied by $P_{n}(\cos \theta)$ and $\left.Q_{n}(\cos \theta)\right)$ by substituting $n=-1 / 2+i q$. The functions

$$
(\cosh \eta-\cos \theta)^{1 / 2} L_{q}(-\cos \theta) \cos q \eta
$$

are harmonic. They are called the conal functions.

As to the convergence of the integral (1) we note that

$$
L_{q}(-\cos \theta) / L_{q}\left(-\cos \theta_{0}\right)
$$

is a positive quantity less than 1 and that

$$
\int_{-\infty}^{\infty} \frac{L_{q}\left(\cos \theta_{0}\right)}{\cosh q \pi} d q
$$

is a convergent integral.

For the capacity of the spindle we obtain

$$
\begin{aligned}
C & =a \int_{-\infty}^{\infty} \frac{L_{q}\left(\cos \theta_{0}\right)}{L_{q}\left(-\cos \theta_{0}\right)} \frac{d q}{\cosh q \pi} \\
& =a \int_{-\infty}^{\infty} \frac{F\left(1 / 2+i q, 1 / 2-i q ; 1 ; \cos ^{2}\left(\theta_{0} / 2\right)\right)}{F\left(1 / 2+i q, 1 / 2-i q ; 1 ; \sin ^{2}\left(\theta_{0} / 2\right)\right)} \frac{d q}{\cosh q \pi} .
\end{aligned}
$$


For $\theta_{0}=\pi / 2$ (sphere) we have $C=a$, since

$$
\int_{-\infty}^{\infty} \frac{d q}{\cosh q \pi}=1
$$

Another form of the potential $u$, involving an infinite series instead of an integral, is due to H. M. Macdonald [10].

16. Example 4. Two spheres. We consider two separated spheres (W. Thomson [35, pp. 86-97]; C. Neumann [14]; Maxwell [11, pp. 266-273]). In this case it is convenient to use again dipolar co-ordinates. But instead of the conal functions we employ now the harmonics

$$
(\cosh \eta-\cos \theta)^{1 / 2} P_{n}(\cos \theta) e^{ \pm(n+1 / 2) \eta}
$$

where $P_{n}$ denotes Legendre's polynomials.

We can represent the two spheres in the form $\eta=\alpha<0$ and $\eta=\beta>0$. In the exterior space $\alpha<\eta<\beta$. Using the harmonics mentioned, we have for the potential function

$$
\begin{aligned}
& u(p)=(2 \cosh \eta-2 \cos \theta)^{1 / 2} \\
& \cdot \sum_{n=0}^{\infty}\left(e^{N \alpha} \frac{e^{N(\beta-\eta)}-e^{N(\eta-\beta)}}{e^{N \delta}-e^{-N \delta}}+e^{-N \beta} \frac{e^{N(\eta-\alpha)}-e^{N(\alpha-\eta)}}{e^{N \delta}-e^{-N \delta}}\right) P_{n}(\cos \theta), \\
& N=n+1 / 2, \quad \delta=\beta-\alpha,
\end{aligned}
$$

and for the capacity

$$
C=2 a \sum_{n=0}^{\infty}\left(e^{N \alpha} \frac{e^{N \beta}-e^{-N \beta}}{e^{N \delta}-e^{-N \delta}}+e^{-N \beta} \frac{e^{-N \alpha}-e^{N \alpha}}{e^{N \delta}-e^{-N \delta}}\right) .
$$

An interesting limiting case arises when the spheres are tangent to each other (Maxwell [11, pp. 273-276]). Denoting their radii by $r$ and $r^{\prime}$, we have in this case

$$
C=\frac{r r^{\prime}}{r+r^{\prime}}\left(-\frac{\Gamma^{\prime}}{\Gamma}\left(\frac{r}{r+r^{\prime}}\right)-\frac{\Gamma^{\prime}}{\Gamma}\left(\frac{r^{\prime}}{r+r^{\prime}}\right)-2 \gamma\right),
$$

where $\Gamma$ denotes Euler's gamma function and $\boldsymbol{\gamma}$ Euler's constant. For $r=r^{\prime}$ we have $C=2 r \log 2=1.39 r$.

Let us consider the generalized condenser in the sense of $\$ 6$ bounded by the first sphere $s_{1}$, the second sphere $s_{2}$, and the sphere of infinitely large radius $s_{0}$. Then the potential functions $u_{1}(p)$ and $u_{2}(p)$ of $\$ 6$ can be expressed in the form 


$$
\begin{aligned}
& u_{1}(p)=(2 \cosh \eta-2 \cos \theta)^{1 / 2} \sum_{n=0}^{\infty} e^{N \alpha} \frac{e^{N(\beta-\eta)}-e^{N(\eta-\beta)}}{e^{N \delta}-e^{-N \delta}} P_{n}\left(\cos ^{\prime} \theta\right), \\
& u_{2}(p)=(2 \cosh \eta-2 \cos \theta)^{1 / 2} \sum_{n=0}^{\infty} e^{-N \beta} \frac{e^{N(\eta-\alpha)}-e^{N(\alpha-\eta)}}{e^{N \delta}-e^{-N \delta}} P_{n}(\cos \theta) .
\end{aligned}
$$

The capacity coefficients $C_{11}, C_{12}=C_{21}, C_{22}$ can also be computed (Maxwell [11, p. 271]).

17. Example 5. Lens. (a) In this case we return again to the peripolar (toroidal) coordinates defining the boundary of the lens by the conditions $\theta=\theta_{1}$ and $\theta=\theta_{2}$. We can always assume that the "upper" surface of the lens is in the upper half-space, $0<\theta_{1}<\pi$. The "lower" surface may be in the upper or lower half-space, $\theta_{1}<\theta_{2}<2 \pi$. In the space exterior to the lens we define $\theta$ according to the condition

$$
\theta_{2}-2 \pi<\theta<\theta_{1},
$$

the value $\theta=0$ corresponding to the point at infinity.

We employ the harmonic functions

$$
(\cosh \eta-\cos \theta)^{1 / 2} L_{q}(-\cosh \eta)\left\{\begin{array}{l}
\cosh q \theta, \\
\sinh q \theta
\end{array}\right.
$$

where $L_{q}(-\cosh \eta)$ is a function arising from the conal function $L_{q}(-\cos \theta)$ by a proper analytic continuation. We mention the following representation:

$$
L_{q}(-\cosh \eta)
$$

$$
=\pi^{-1} \cosh q \pi \int_{-\infty}^{\infty}\left(2 \cosh \eta^{\prime}+2 \cosh \eta\right)^{-1 / 2} \cos q \eta^{\prime} d \eta^{\prime}, q \text { and } \eta \text { real. }
$$

It can be shown that for $q \rightarrow \pm \infty, \eta$ fixed,

$$
L_{q}(-\cosh \eta)=O\left(e^{\epsilon|q|}\right) \text {, }
$$

where $\epsilon$ is an arbitrary positive number independent of $q$.

Now we have for the potential function (Mehler $[12, \mathrm{p} .141]$ )

$$
\begin{aligned}
& u(p)=(2 \cosh \eta-2 \cos \theta)^{1 / 2} \\
& \text { (5) } \quad \int_{-\infty}^{\infty} \frac{\sinh q\left(\theta_{1}-\theta\right) \cosh q\left(\pi-\theta_{2}\right)+\cosh q\left(\pi-\theta_{1}\right) \sinh q\left(2 \pi-\theta_{2}+\theta\right)}{2 \sinh q\left(2 \pi+\theta_{1}-\theta_{2}\right) \cosh q \pi} \\
& \cdot L_{q}(-\cosh \eta) d q .
\end{aligned}
$$

For the capacity we find 
(6)

$$
\begin{aligned}
C & =a \int_{-\infty}^{\infty} \frac{\sinh q \theta_{1} \cosh q\left(\pi-\theta_{2}\right)+\cosh q\left(\pi-\theta_{1}\right) \sinh q\left(2 \pi-\theta_{2}\right)}{\sinh q\left(2 \pi+\theta_{1}-\theta_{2}\right) \cosh q \pi} d q \\
& =a \int_{-\infty}^{\infty} \frac{\sinh q\left(\pi+\theta_{1}-\theta_{2}\right)+\cosh q\left(2 \pi-\theta_{1}-\theta_{2}\right) \sinh q \pi}{\sinh q\left(2 \pi+\theta_{1}-\theta_{2}\right) \cosh q \pi} d q .
\end{aligned}
$$

As to the convergence of (5) and (6) we observe that for $q \rightarrow+\infty$ the order of magnitude of the factor of $L_{q}$ on the range (1) is less than

$$
e^{-q\left(\pi-\left|\pi-\theta_{2}\right|\right)}+e^{-q \theta_{1}},
$$

and this expression approaches 0 exponentially. Now we use (4).

(b) The following special cases of the preceding general result are of particular interest.

( $\alpha$ ) Spherical bowl (calotte), $\theta_{1}=\theta_{2}, 0<\theta_{1}<\pi$ (W. Thomson [35, $\mathrm{p}$. 186]; C. Neumann [17]); the capacity is

$$
\begin{aligned}
C & =a \int_{-\infty}^{\infty}\left(\frac{\cosh q\left(\pi-\theta_{1}\right)}{\cosh q \pi}\right)^{2} d q \\
& =\frac{a}{\pi}+\left(1-\frac{\theta_{1}}{\pi}\right) \frac{a}{\sin \theta_{1}}=\frac{a+a^{\prime}}{\pi},
\end{aligned}
$$

where $a$ is the radius of the "rim" and $a^{\prime}$ the "spherical radius" of the bowl.

( $\beta$ ) Circular disk, limiting case of $(\alpha), \theta_{1}=\theta_{2} \rightarrow \pi ; C=2 a / \pi .^{6}$

$(\gamma)$ Half spherical shell (thin), special case of $(\alpha), \theta_{1}=\theta_{2}=\pi / 2$; $C=a(1 / \pi+1 / 2)$.

( $\delta$ ) Half spherical solid, $\theta_{1}=\pi / 2, \theta_{2}=\pi ; C=2 a\left(1-3^{-1 / 2}\right)$.

(є) Symmetrical lens, $\theta_{1}+\theta_{2}=2 \pi$; in this case

$$
C=a \int_{-\infty}^{\infty} \frac{\cosh q\left(\pi-\theta_{1}\right)}{\cosh q \theta_{1} \cosh q \pi} d q .
$$

(§) Sphere, $\theta_{2}-\theta_{1}=\pi$;

$$
C=a \int_{-\infty}^{\infty} \frac{\cosh q\left(\pi-2 \theta_{1}\right)}{\cosh q \pi} d q=\frac{a}{\sin \theta_{1}} .
$$

$(\eta)$ Two spheres tangent to each other, a suitable limiting case, $\theta_{1} \rightarrow 0, \theta_{2} \rightarrow 2 \pi, a \rightarrow 0$; cf. $\$ 16$.

$(\theta)$ The two spheres forming the lens are orthogonal in such a way

- According to a remark of W. Thomson [35, p. 180] Cavendish found by experiment $a / C=1.571$. 
that the exterior (dielectric) angle is $\pi / 2, \theta_{2}-\theta_{1}=3 \pi / 2$ (Maxwell [11, p. 261]);

$$
C=r+r^{\prime}-a,
$$

where $r$ and $r^{\prime}$ are the radii of the spheres.

The capacity integral (6) can be computed in closed form in all cases when the dielectric angle $2 \pi+\theta_{1}-\theta_{2}$ is a rational multiple of $\pi$. As to the potential function $u(p)$, it can be computed in terms of elementary functions only for certain special multiples; in other cases it leads to elliptic and more complicated functions. (See Macdonald [9].)

In certain cases the problem of the lens can be also treated by using the famous method of electric images due to $W$. Thomson $[35, \mathrm{pp}$. 144-191]; see also Maxwell [11].

\section{BIBLIOGRAPHY}

1. K. Aichi, Note on the capacity of a nearly spherical conductor and especially of an ellipsoidal conductor, Proceedings of the Physico-Mathematical Society of Tokyo (2) vol. 4 (1908) pp. 243-246.

2. T. Bonnesen and W. Fenchel, Theorie der konvexen Körper, Ergebnisse der Mathematik und ihrer Grenzgebiete, vol. 3, Berlin, 1934.

3. R. Clausius, Die mechanische Behandlung der Electricität, Braunschweig, 1879.

4. G. Faber, Beweis, dass unter allen homogenen Membranen von gleicher Fläche und gleicher Spannung die kreisförmige den tiefsten Grundton gibt, Sitzungsberichte der Bayrischen Akademie der Wissenschaften (1923) pp. 169-172.

5. E. Heine, Handbuch der Kugelfunctionen, Theorie und Aniwendungen, vol. 2, 2nd ed., Berlin, 1881.

6. W. M. Hicks, On toroidal functions, Philos. Trans. Roy. Soc. London, Ser. A. vol. 172 (1881) pp. 609-652.

7. E. W. Hobson, The theory of spherical and ellipsoidal harmonics, Cambridge, 1931.

8. O. D. Kellogg, Foundations of potential theory, Berlin, 1929.

9. H. M. Macdonald, The electrical distribution on a conductor bounded by two spherical surfaces cutting at any angle, Proc. London Math. Soc. vol. 26 (1895) pp. 156172.

10. - Demonstration of Green's formula for electric density near the vertex of a right cone, Memoirs presented to the Cambridge Philosophical Society on the occasion of the jubilee of Sir George Gabriel Stokes, Cambridge, 1900, pp. 292-297.

11. J. C. Maxwell, A treatise on electricity and magnetism, vol. 1, 3d ed., Oxford, 1892.

12. F. G. Mehler, Ueber die Vertheilung der statischen Elektricität in einem von zwei Kugelkalotten begrenzten Körper, J. Reine Angew. Math. vol. 68 (1868) pp. 134-150.

13. - Ueber eine mit den Kugel- und Cylinderfunctionen verwandte Function und ihre Anwendung in der Theorie der Elektricitätsvertheilung, Math. Ann. vol. 18 (1881) pp. 161-194.

14. C. Neumann, Ueber das Gleichgewicht der Wärme und das der Elektricität in einem Körper, welcher von zwei nicht concentrischen Kugelflächen begrenzt wird, J. Reine Angew. Math. vol. 62 (1862) pp. 36-49. 
15

15. - Theorie der Elektricitäts- und Wärmevertheilung in einem Ringe, Halle, 1864.

16. - Über die peripolaren Koordinaten, Abhandlungen der mathematischphysischen Classe der königl. sächsischen Gesellschaft der Wissenschaften vol. 12 (1880) pp. 365-398.

17. - Die Vertheilung der Elektricität auf einer Kugelcalotte, Abhandlungen der mathematisch-physischen Classe der königl. sächsischen Gesellschaft der Wissenschaften vol. 12 (1880) pp. 401-456.

18. - Ueber die Mehler'schen Kegelfunctionen und deren Anwendung auf elektrostatische Probleme, Math. Ann. vol. 18 (1881) pp. 195-236.

19. W. D. Niven, On the harmonics of a ring. Proc. London Math. Soc. (2) vol. 24 (1892) pp. 372-386.

20. H. Poincaré, Figures d'équilibre d'une masse fluide, Paris, 1903.

21. G. Polya, Problem, Archiv der Mathematik und Physik (3) vol. 26 (1917) p. 65 ; solution by G. Szegö, vol. 28 (1920) pp. 81-82.

22. - Beitrag zur Verallgemeinerung des Verzerrungssatzes auf mehrfach zusammenhängende Gebiete. Zweite Mitteilung, Preuss. Akad. Wiss. Sitzungsber. (1928) pp. 280-282.

23. G. Pollya and G. Szegö, Aufgaben und Lehrsätze aus der Analysis, vols. 1 and 2, Berlin, 1925.

24. - - Über den transfiniten Durchmesser (Kapazitätskonstante) von ebenen und räumlichen Punktmengen, J. Reine Angew. Math. vol. 165 (1931) pp. 4-49.

25. - Inequalities for the capacity of a condenser, Amer. J. Math. vol. 67 (1945) pp. 1-32.

26. Lord Rayleigh, On the electrical capacity of approximate spheres and cylinders, Philosophical Magazine vol. 31 (1916) pp. 177-186; Scientific papers, vol. 6, Cambridge, 1920, pp. 383-392.

27. B. Riemann, Ueber das Potential eines Ringes; posthumous paper, Gesammelte Mathematische Werke und Wissenschaftlicher Nachlass, 2d ed., 1892, pp. 431-436.

28. Riemann-Weber (Ph. Frank and R. v. Mises), Die Differential- und Integralgleichungen der Mechanik und Physik, vol. 1, 8th ed., Braunschweig, 1930.

29. A. Russell, The eighth Kelvin lecture. Some aspects of Lord Kelvin's life and work, The Journal of the Institution of Electrical Engineers vol. 55 (1917) pp. 1-17.

30. T. J. Stieltjes, Sur les racines de l'équation $X_{n}=0$, Acta Math. vol. 9 (1886) pp. 385-400. Oeuvres complètes, vol. 2, pp. 73-88.

31. G. Szegö, Verallgemeinerung des ersten Bieberbachschen Flächensatzes auf mehrfach zusammenhängende Gebiete, Preuss. Akad. Wiss. Sitzungsber. (1928) pp. 477481.

32. - Über einige Extremalaufgaben der Potentialtheorie, Math. Zeit. vol. 31 (1930) pp. 583-593.

33. — Über einige neue Extremaleigenschaften der Kugel, Math. Zeit. vol. 33 (1931) pp. 419-425.

34. - An inequality, Quart. J. Math. vol. 6 (1935) pp. 78-79.

35. W. Thomson, Reprint of papers on electrostatics and magnetism, 2nd ed., London, 1884.

36. G. N. Watson, An inequality, Quart. J. Math. vol. 5 (1934) pp. 221-223.

\section{STANFORD UNIVERSITY}

